\title{
ESTUDO CINÉTICO DA DECOMPOSIÇÃO TÉRMICA DOS ÁCIDOS MIRÍSTICO E OLÉICO E DO ÓLEO DE PINHÃO MANSO VIA ANÁLISE TERMOGRAVIMÉTRICA
}

\author{
C. M. T. de ALMEIDA ${ }^{1}$, E. D. V. BRUCE ${ }^{2}$, C. M. B. M. BARBOSA ${ }^{3}$, R. T. F. FRETY ${ }^{4}$, J. G. A. \\ $\mathrm{PACHECO}^{5}$ \\ 1,2,3,4,5 Universidade Federal de Pernambuco, Departamento de Engenharia Química \\ E-mail para contato: camila-mta@ hotmail.com
}

\begin{abstract}
RESUMO - Este trabalho propõe o estudo cinético da decomposição térmica dos ácidos mirístico e oléico, e do óleo de pinhão manso via análise termogravimétrica. Os testes foram realizados em uma termobalança sob fluxo de nitrogênio, utilizando uma faixa de temperatura de $30-900^{\circ} \mathrm{C}$, em diferentes taxas de aquecimento. As curvas TG/DTG mostram que os compostos modelos apresentam uma única etapa de perda de massa, e o óleo de pinhão manso, duas etapas de perda. As curvas DTA demonstram o comportamento endotérmico de parte dos eventos. Os parâmetros cinéticos foram estimados a partir do modelo de cinética livre proposto por Vyazovkin. Os valores obtidos para a energia média de ativação na decomposição térmica completa dos ácidos mirístico e oléico e do óleo de pinhão manso foram, respectivamente, $86,4 \mathrm{~kJ}^{\mathrm{mol}}{ }^{-1} ; 80,1 \mathrm{~kJ} . \mathrm{mol}^{-1} \mathrm{e}$ $132,4 \mathrm{~kJ} \cdot \mathrm{mol}^{-1}$.
\end{abstract}

\section{INTRODUÇÃO}

O aumento na demanda por fontes renováveis de energia, o esgotamento das reservas de petróleo de fácil extração e a crescente preocupação com a preservação ambiental tem incentivado o desenvolvimento de biocombustíveis, conforme citado por Huber et al. (2006).

O uso de oleaginosas que não competem com a alimentação visando a produção de biocombustíveis têm atraído um crescente interesse durante as duas últimas décadas. Segundo Azam et al. (2005), óleos vegetais são constituídos por ácidos graxos ligados através do grupo carboxílico com um esqueleto de glicerol, diferindo na quantidade de carbonos que compõem a cadeia e presença, ou ausência de insaturações. Biswas e Sharma (2013) afirmam que o óleo de pinhão manso (Jatropha curcas), rico em ácidos graxos livres, pode ser considerado uma promissora fonte de biocombustíveis.

Kumar et al. (2010) relatam que os principais problemas associados à utilização de óleos vegetais in natura como combustíveis líquidos são a instabilidade química, alta viscosidade e formação de depósitos de carbono nos motores. Desse modo, o craqueamento de óleos vegetais surge como uma alternativa promissora para a produção de biocombustíveis derivados de oleaginosas. 
A fim de compreender o processo de craqueamento do óleo de pinhão manso, é necessário o conhecimento dos parâmetros cinéticos da reação de decomposição, como a energia de ativação e o fator pré-exponencial de Arrhenius, conforme sugerido por Biswas e Sharma (2013). Segundo Pacheco et al. (2005), ao longo do craquemaneto ocorrem diversas reações paralelas e consecutivas, cuja modelagem cinética convencional apresenta bastante complexidade. Uma forma alternativa é a utilização de modelos de cinética livre, que calcula a energia de ativação efetiva como função da conversão $(\alpha)$ de uma reação, por meio de ensaios dinâmicos via análise termogravimétrica.

\section{METODOLOGIA}

\subsection{Reagentes e Equipamento}

O óleo ácido de pinhão manso foi fornecido pela empresa Bioauto. Seu índice de acidez foi determinado segundo a norma ASTM D664, obtendo-se o valor de 20,4 mg de $\mathrm{KOH} / \mathrm{g}$ de óleo. Os compostos modelos, ácidos mirístico e oléico, foram obtidos por meio da empresa Sigma-Aldrich, ambos com pureza de $99 \%$.

Os reagentes foram submetidos a testes termogravimétricos para obtenção das faixas de perdas de massa. Foi utilizada uma termobalança Perkin Elmer, modelo STA 6000. Os testes dinâmicos foram realizados sob fluxo de nitrogênio $\left(20 \mathrm{~mL} \cdot \mathrm{min}^{-1}\right)$, utilizando uma faixa de temperatura de 30 $900{ }^{\circ} \mathrm{C}$, em diferentes taxas de aquecimento $\left(5,10,30\right.$ e $\left.40{ }^{\circ} \mathrm{C} \cdot \mathrm{min}^{-1}\right)$. A massa de reagente foi fixada em $10 \mathrm{mg}$.

\subsection{Modelo de Cinética Livre de Viazovkin}

Segundo Ramani et al. (2010), a velocidade de uma reação depende da extensão da conversão $(\alpha)$, temperatura $(\mathrm{T})$ e tempo $(\mathrm{t})$. Essa dependência é expressa conforme Equação 1, onde k(T) é a constante de conversão e $\mathrm{f}(\alpha)$ é a função da reação.

$$
\frac{d \alpha}{d t}=k(T) f(\alpha) \quad(E q \cdot 1)
$$

A conversão é determinada por meio da termogravimetria, acompanhando a perda de massa em função do tempo. A constante de reação depende da temperatura e é dada em termos da Equação 2, onde $\mathrm{R}$ é a constante universal dos gases, A o fator pré-exponencial de Arrhenius e $\mathrm{E}$ a energia de ativação efetiva.

$$
k(T)=A e^{\left(\frac{-E}{R T}\right)} \quad(E q \cdot 2)
$$

Substituindo a Equação 2 na Equação 1, obtem-se a Equação 3.

$$
\frac{d \alpha}{d t}=A e^{\left(\frac{-E}{R T}\right)} f(\alpha)
$$


A taxa de aquecimento é dada por ( $\beta=\mathrm{dT} / \mathrm{dt})$. Aplicando a integração por separação de variáveis a Equação 3 e em seguida tomando o logaritmo neperiano desse resultado, obtém-se a Equação 4.

$$
\ln \left(\frac{\beta}{T_{\alpha}^{2}}\right)=\ln \left(\frac{A R}{E_{\alpha} g(\alpha)}\right)-\frac{E_{\alpha}}{R T_{\alpha}}
$$

De acordo com Viazovkin e Sbirrazzuoli (2006), o modelo de cinética livre permite a avaliação dos parâmetros cinéticos sem a necessidade de um modelo matemático típico para a cinética da reação. Assim, a decomposição térmica é realizada a diferentes taxas de aquecimento $(\beta)$ e as curvas de conversão são calculadas a partir dos dados termogravimétricos. Para cada $\alpha, \ln \left(\beta / \mathrm{T}_{\alpha}{ }^{2}\right)$ são plotados versus $\left(1 / \mathrm{T}_{\alpha}\right)$, ajustando-se a uma linha cujo coeficiente angular é igual a $\left(-\mathrm{E}_{\alpha} / \mathrm{R}\right)$.

\section{RESULTADOS E DISCUSSÃO}

A Figura 1 apresenta as curvas termogravimétricas, TG e DTG, de perda de massa dos ácidos mirístico e oléico e do óleo de pinhão manso, em diferentes taxas de aquecimento.
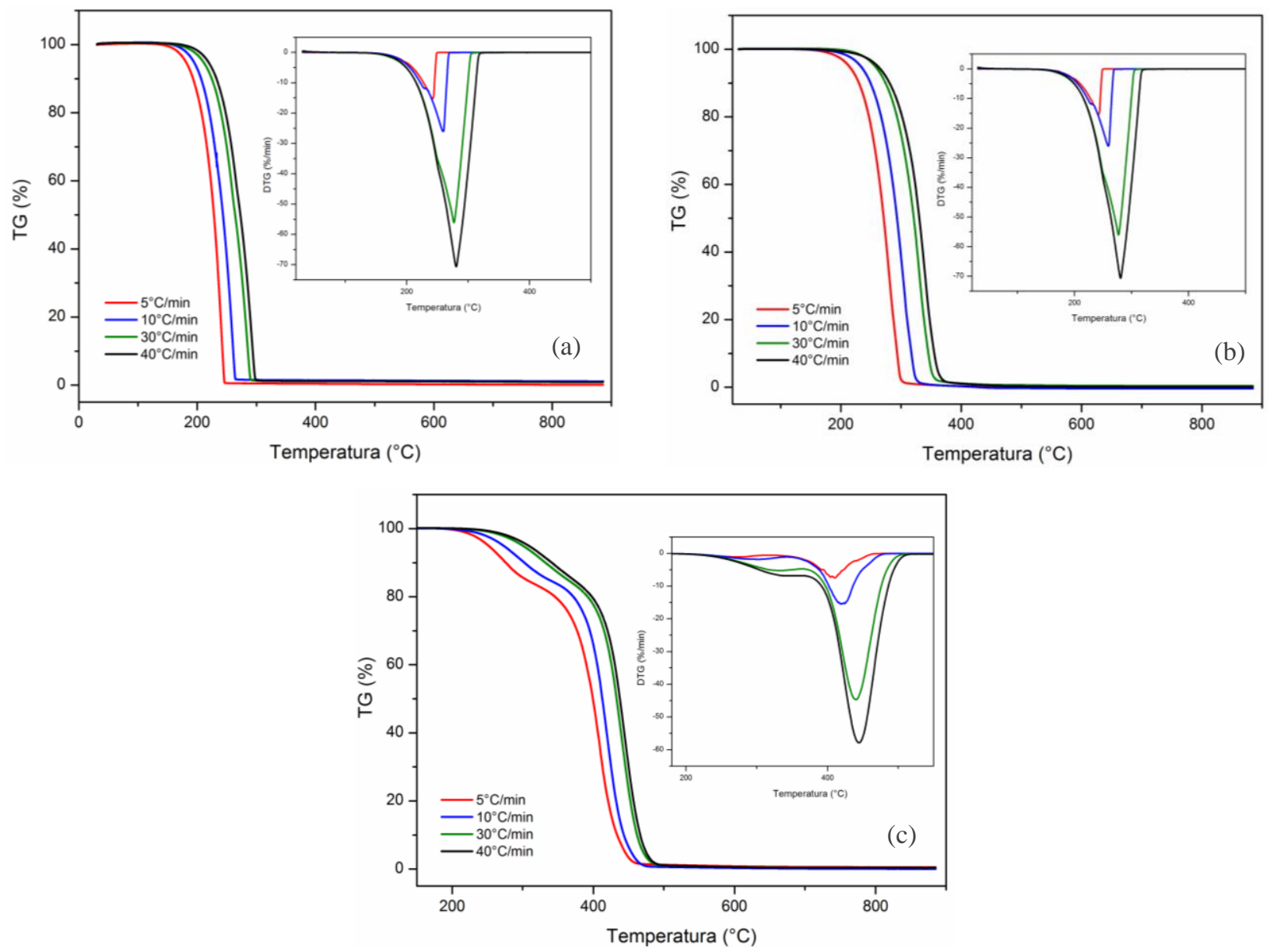

Figura 1 - Curvas TG e DTG do: (a) ácido mirístico, (b) ácido oléico e (c) óleo de pinhão manso. 
Os compostos modelos apresentam um intervalo de perda de massa, conforme pode ser visualizado nas curvas DTG da Figura 1 (a) e (b). Biswas e Sharma, estudaram as perdas de massa do óleo de pinhão manso, que ocorrem em duas etapas, e analisaram os resultados como sendo devido a quebra das moléculas de óleo em compostos orgânicos de menor tamanho. Por analogia, as perdas de massa da Figura 1 foram atribuídas a decomposição dos ácidos mirístico e oléico, em produtos de craqueamento. Biswas e Sharma (2013) sugerem que a primeira etapa é devido a quebra de triglicerídeos maiores em moléculas orgânicas menores, e a segunda etapa corresponde a desvolatilização total das moléculas orgânicas.

As características das curvas de DTG da decomposição térmica dos reagentes em estudo estão na Tabela 1. Observa-se que quanto maior for a taxa de aquecimento utilizada, maior será a faixa de temperatura requerida para uma completa perda de massa.

Tabela 1 - Características das curvas DTG na decomposição térmica dos reagentes em estudo

\begin{tabular}{|c|c|c|c|c|c|}
\hline \multirow{2}{*}{ Reagentes } & \multirow{2}{*}{$\begin{array}{c}\beta \\
\left({ }^{\circ} \mathrm{C} / \mathrm{min}\right)\end{array}$} & \multicolumn{4}{|c|}{ Temperatura $\left({ }^{\circ} \mathrm{C}\right)$} \\
\hline & & $\mathrm{T}_{\text {início }}$ & $\mathrm{T}_{\max 1}$ & $\mathrm{~T}_{\max 2}$ & $\mathrm{~T}_{\text {fim }}$ \\
\hline \multirow{4}{*}{$\begin{array}{l}\text { Ácido } \\
\text { Mirístico }\end{array}$} & 5 & 160 & 242 & - & 250 \\
\hline & 10 & 162 & 259 & - & 270 \\
\hline & 30 & 163 & 277 & - & 307 \\
\hline & 40 & 161 & 281 & - & 324 \\
\hline \multirow{4}{*}{$\begin{array}{l}\text { Ácido } \\
\text { Oléico }\end{array}$} & 5 & 170 & 281 & - & 329 \\
\hline & 10 & 173 & 304 & - & 346 \\
\hline & 30 & 173 & 329 & - & 392 \\
\hline & 40 & 170 & 338 & - & 496 \\
\hline \multirow{4}{*}{$\begin{array}{c}\text { Óleo Pinhão } \\
\text { Manso }\end{array}$} & 5 & 187 & 271 & 410 & 469 \\
\hline & 10 & 190 & 289 & 420 & 487 \\
\hline & 30 & 192 & 321 & 440 & 507 \\
\hline & 40 & 195 & 331 & 444 & 513 \\
\hline
\end{tabular}

A Figura 2 apresenta as curvas de análise térmica diferencial (DTA) dos compostos modelos e do óleo em estudo, realizadas com taxa de aquecimento de $30^{\circ} \mathrm{C} \cdot \mathrm{min}^{-1}$. A curva DTA do ácido mirístico apresenta dois picos endotérmicos. O primeiro pico é relativo ao processo físico de fusão $\left(\mathrm{T}_{\text {fusão: }}: 54^{\circ} \mathrm{C}\right)$, fato evidenciado pela ausência de perda de massa nessa região. $\mathrm{O}$ segundo pico apresenta característica predominantemente endotérmica, podendo está associado aos processos de volatilização $\left(\mathrm{T}_{\text {vaporização: }} 250^{\circ} \mathrm{C}\right)$ e, em menor escala, craqueamento.

De modo semelhante, o ácido oléico apresenta em sua curva DTA um pico endotérmico. O mesmo pode ser associado à volatilização $\left(\mathrm{T}_{\text {vaporização }}: 360^{\circ} \mathrm{C}\right)$ parcial desse composto modelo, assim como, a ocorrência de reações de craqueamento que acontecem paralelamente.

O óleo de pinhão manso apresenta em sua curva DTA dois eventos com características 
endotérmicas. Os picos observados podem está associados ao processo de volatilização de alguns ácidos graxos que compõem o óleo em estudo, além da ocorrência de reações de craqueamento.

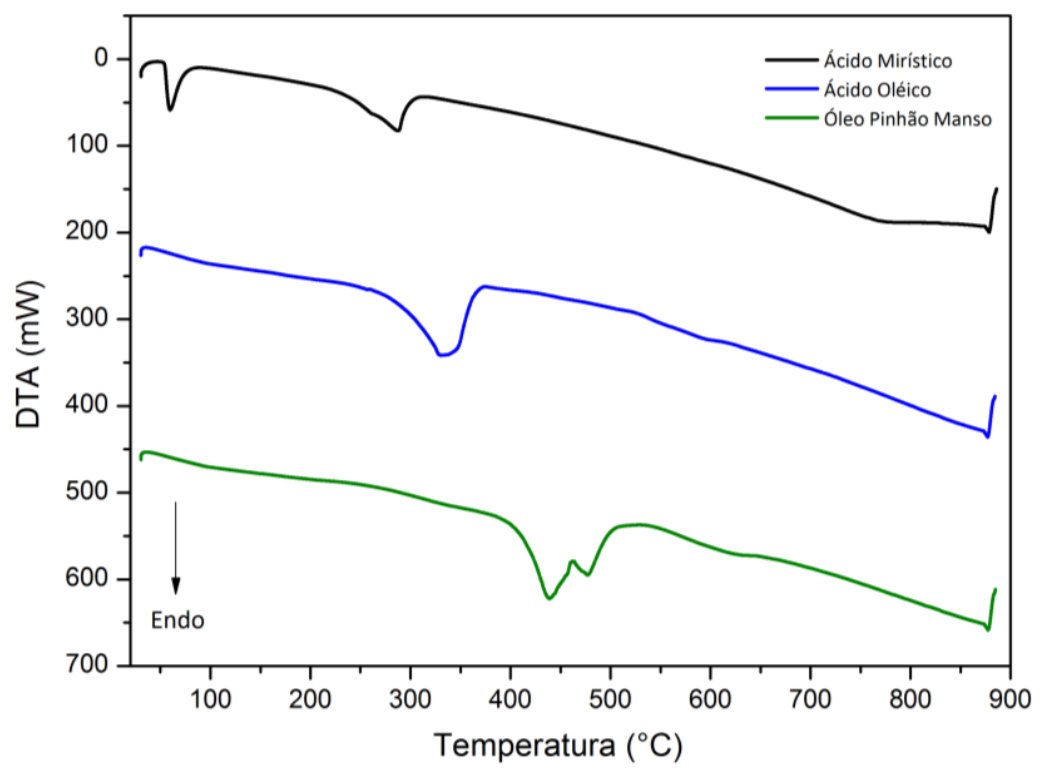

Figura 2 - Curvas DTA dos compostos modelos e do óleo de pinhão manso utilizando taxa de aquecimento de $30^{\circ} \mathrm{C} \cdot \mathrm{min}^{-1}$.

A Tabela 2 apresenta as principais características das curvas DTA da Figura 2, acompanhando as perdas de massa dos reagentes em estudo, a taxa de aquecimento de $30^{\circ} \mathrm{C} \cdot \mathrm{min}^{-1}$.

Tabela 2 - Características das curvas DTA

\begin{tabular}{|c|c|c|c|c|c|}
\hline Reagente & Pico & $\begin{array}{c}\mathrm{T}_{\text {pico }} \\
\left({ }^{\circ} \mathrm{C}\right)\end{array}$ & $\begin{array}{c}\Delta \mathrm{H} \\
(\mathrm{J} / \mathrm{g})\end{array}$ & Transformação & Atribuição \\
\hline \multirow{2}{*}{$\mathrm{AM}$} & 1 & 60 & 161 & Endotérmica & Fusão \\
\cline { 2 - 6 } & 2 & 287 & 315 & Endotérmica & Craqueamento/Volatilização \\
\hline \multirow{2}{*}{$\mathrm{AO}$} & 1 & 331 & 257 & Endotérmica & Craqueamento/Volatilização \\
\hline \multirow{2}{*}{$\mathrm{PM}$} & 1 & 439 & 73 & Endotérmica & Craqueamento/Volatilização \\
\cline { 2 - 6 } & 2 & 478 & 10 & Endotérmica & Craqueamento/Volatilização \\
\hline
\end{tabular}

Os parâmetros cinéticos desse trabalho foram estimados a partir do modelo de cinética livre de Vyazovkin, conforme retas isoconvercionais da Figura 3. A energia de ativação depende do grau de conversão $(\alpha)$, que variou de 10 a $90 \%$ para os compostos modelos e de, 1 a $90 \%$ para o óleo de pinhão manso. A energia de ativação efetiva $\left(\mathrm{E}_{\alpha}\right)$ e o fator pré-exponencial de Arrhenius (A) foram obtidos a partir dos coeficientes angular e linear, respectivamente, das retas. Os valores médios de $\mathrm{E}_{\alpha}$ e A foram $87,8 \mathrm{~kJ} \mathrm{~mol}^{-1}$ e $1,1 \times 10^{9} \mathrm{~K}$, para a decomposição térmica do ácido mirístico e $80,7 \mathrm{~kJ} \mathrm{~mol}^{-1} \mathrm{e}$ $2,2 \times 10^{7} \mathrm{~K}$, para a decomposição do ácido oléico. 
A Figura 3 (c) apresenta duas regiões. A primeira, $1 \%<\alpha<10 \%$, é atribuída a quebra de moléculas grandes de triglicerídeos em moléculas menores, e a segunda região, $20 \%<\alpha<90 \%$, a completa degradação das moléculas orgânicas menores. A energia média de ativação para a primeira região foi $77,8 \mathrm{~kJ} \cdot \mathrm{mol}^{-1}$ e para a segunda, $186,9 \mathrm{~kJ} \cdot \mathrm{mol}^{-1}$. A energia média de ativação calculada para a decomposição completa do óleo foi $132,4 \mathrm{~kJ}_{\text {. }} \mathrm{mol}^{-1}$, valor esse próximo ao encontrado por Biswas e Sharma (2013) que foi de 168,18 $\mathrm{kJ} \mathrm{mol}^{-1}$. Vale ressaltar que Biswas e Sharma (2013) utilizaram o modelo cinético de Friedman, enquanto que nesse trabalho foi utilizado o modelo cinético de Vyazovkin, fato que justifica a pequena diferença nos valores das energias médias de ativação.

O fator pré-exponencial médio de Arrhenius calculado para a primeira região de degradação do óleo de pinhão manso foi $5,3 \times 10^{7} \mathrm{~K}$ e para a segunda região, $1,0 \times 10^{16} \mathrm{~K}$. O valor médio global calculado foi de $5,0 \times 10^{15} \mathrm{~K}$.
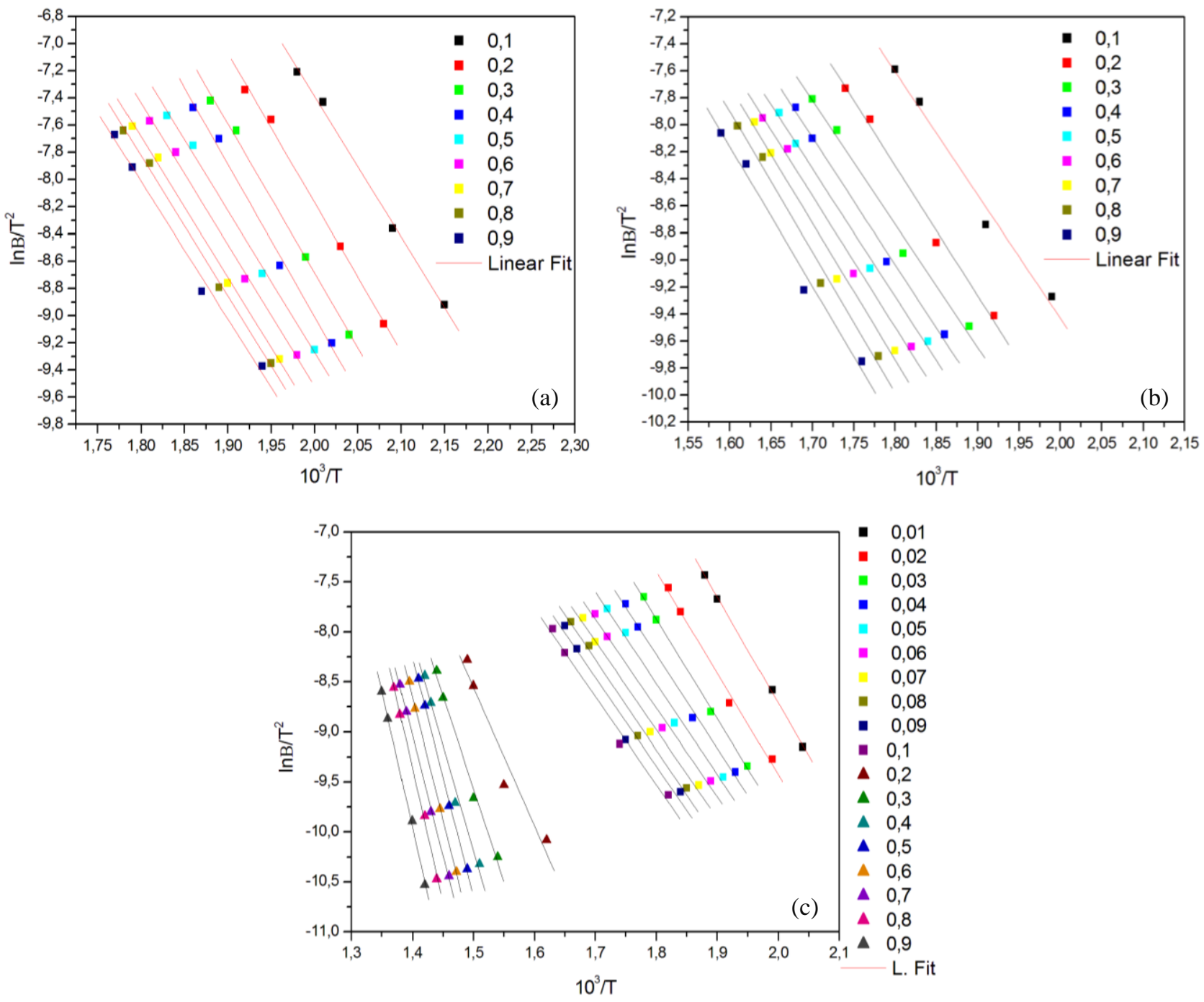

Figura 3 - Retas isoconvercionais do (a) ácido mirístico, (b) ácido oléico e (c) óleo de pinhão manso. 
A Tabela 3 apresenta os parâmetros cinéticos obtidos por meio das retas isoconvercionais da Figura 3, para a perda de massa dos ácidos mirístico e oléico e o óleo de pinhão manso.

Tabela 3 - Parâmetros cinéticos dos reagentes em estudo

\begin{tabular}{|c|c|c|c|c|c|c|}
\hline \multirow{2}{*}{$\begin{array}{c}\alpha \\
(\%)\end{array}$} & \multicolumn{2}{|c|}{ Ácido Mirístico } & \multicolumn{2}{c|}{ Ácido Oléico } & \multicolumn{2}{c|}{ Óleo Pinhão Manso } \\
\cline { 2 - 7 } & $\begin{array}{c}\mathrm{E}_{\alpha} \\
\left(\mathrm{kJ}^{-} \mathrm{mol}^{-1}\right)\end{array}$ & $\begin{array}{c}\mathrm{A} \\
(\mathrm{K})\end{array}$ & $\begin{array}{c}\mathrm{E}_{\alpha} \\
\left(\mathrm{kJ}^{-} \mathrm{mol}^{-1}\right)\end{array}$ & $\begin{array}{c}\mathrm{A} \\
(\mathrm{K})\end{array}$ & $\begin{array}{c}\mathrm{E}_{\alpha} \\
\left(\mathrm{kJ}^{-} \mathrm{mol}^{-1}\right)\end{array}$ & $\begin{array}{c}\mathrm{A} \\
(\mathrm{K})\end{array}$ \\
\hline 1,0 & - & - & - & - & 88,1 & $2,8 \times 10^{7}$ \\
\hline 2,0 & - & - & - & - & 84,5 & $1,1 \times 10^{7}$ \\
\hline 3,0 & - & - & - & - & 82,8 & $6,9 \times 10^{6}$ \\
\hline 4,0 & - & - & - & - & 78,1 & $2,2 \times 10^{6}$ \\
\hline 5,0 & - & - & - & - & 75,8 & $1,2 \times 10^{6}$ \\
\hline 6,0 & - & - & - & - & 73,8 & $7,2 \times 10^{5}$ \\
\hline 7,0 & - & - & - & - & 73,6 & $6,5 \times 10^{5}$ \\
\hline 8,0 & - & - & - & - & 74,9 & $8,3 \times 10^{5}$ \\
\hline 9,0 & - & - & - & - & 73,7 & $5,9 \times 10^{5}$ \\
\hline 10,0 & 86,1 & $6,3 \times 10^{8}$ & 75,9 & $6,2 \times 10^{6}$ & 73,3 & $5,0 \times 10^{5}$ \\
\hline 20,0 & 91,2 & $2,1 \times 10^{9}$ & 80,1 & $1,6 \times 10^{7}$ & 114,6 & $5,1 \times 10^{8}$ \\
\hline 30,0 & 91,2 & $1,9 \times 10^{9}$ & 76,0 & $6,1 \times 10^{6}$ & 154,6 & $5,1 \times 10^{11}$ \\
\hline 40,0 & 93,2 & $3,0 \times 10^{9}$ & 78,1 & $9,8 \times 10^{6}$ & 175,5 & $1,8 \times 10^{13}$ \\
\hline 50,0 & 86,7 & $5,6 \times 10^{8}$ & 78,6 & $1,1 \times 10^{7}$ & 198,3 & $9,9 \times 10^{14}$ \\
\hline 60,0 & 86,5 & $4,9 \times 10^{8}$ & 80,6 & $1,7 \times 10^{7}$ & 203,8 & $2,1 \times 10^{15}$ \\
\hline 70,0 & 85,9 & $3,9 \times 10^{8}$ & 84,0 & $3,3 \times 10^{7}$ & 199,2 & $7,4 \times 10^{14}$ \\
\hline 80,0 & 85,7 & $3,7 \times 10^{8}$ & 86,6 & $5,3 \times 10^{7}$ & 223,3 & $3,9 \times 10^{16}$ \\
\hline 90,0 & 84,1 & $2,4 \times 10^{8}$ & 86,1 & $4,2 \times 10^{7}$ & 225,6 & $3,7 \times 10^{16}$ \\
\hline
\end{tabular}

\section{CONCLUSÕES}

A partir dos dados obtidos nas curvas de TG/DTG, constata-se que a degradação térmica do óleo de pinhão manso é um processo complexo, que ocorre em duas etapas, onde a primeira é devida a quebra de triglicerídeos maiores em moléculas orgânicas menores, e a segunda corresponde a volatilização e craqueamento das moléculas orgânicas. De forma semelhante, os compostos modelos de ácidos graxos apresentaram uma única etapa de perda de massa, atribuída a volatilização e craqueamento dos mesmos.

Com base nas curvas DTA, observou-se a presença de dois picos endotérmicos ao longo da degradação térmica do ácido mirístico, onde o primeiro pico é relativo a fusão e o segundo aos processos de volatilização e craqueamento. De modo análogo, o ácido oléico apresentou um pico endotérmico associado à sua parcial volatilização e craqueamento. $\mathrm{O}$ óleo de pinhão manso 
apresentou dois picos endotérmico, que podem está associados a reações craqueamento e ao processo de volatilização de ácidos graxos.

Os parâmetros cinéticos da perda de massa dos reagentes desse trabalho foram determinados a partir do modelo de cinética livre de Vyazovkin. Os valores médios de $\mathrm{E}_{\alpha} \mathrm{e} \mathrm{A}$ calculados para o ácido mirístico, ácido oléico e o óleo de pinhão manso foram, respectivamente, $87,8 \mathrm{~kJ}^{\mathrm{mol}}{ }^{-1}$ e $1,1 \times 10^{9} \mathrm{~K}$; $80,7 \mathrm{~kJ} \cdot \mathrm{mol}^{-1}$ e $2,2 \times 10^{7} \mathrm{~K} ; 132,4 \mathrm{~kJ} \cdot \mathrm{mol}^{-1}$ e $5,0 \times 10^{15} \mathrm{~K}$.

\section{AGRADECIMENTOS}

Os autores deste trabalho agradecem ao financiamento do Programa de Recursos Humanos em Petróleo e Gás Natural (PRH-28), à Fundação de Amparo à Ciência e Tecnologia de Pernambuco (FACEPE), ao Laboratório de Tecnologias e Processos Limpos (LateClim), ao Departamento de Engenharia Química (DEQ) e a Universidade Federal de Pernambuco (UFPE).

\section{REFERÊNCIAS}

BISWAS, S.; SHARMA, D. Studies on cracking of Jatropha oil. J. Anal. Appl. Pyrolysis, v. 99, p. 122-129, 2013.

HUBER, G.; IBORRA, S.; CORMA A. Synthesis of transportation fuels from biomass: chemistry, catalyst and engineering. Chem. Rev., v. 106, p. 4044-4098, 2006.

KUMAR, R.; RANA, B.; TIWARI, R.; VERMA, D.; KUMAR, R.; JOSHI, R.; GARG, M.; SINHA, A.; Hydroprocessing of jatropha oil and its mixtures with gas oil, Green Chem., v. 12, p. 2232-2239, 2010.

PACHECO, J.; CRUZ, E.; SILVA, A.; SOUZA, M.; ARAUJO, A. Thermo gravimetric kinetics of polypropylene degradation on ZSM-12 and ZSM-5 catalysts. Catal. Today, v. 107-108, p. 507-512, 2005.

RAMANI, R.; SRIVATAVA, J. Application of model-free kinetics to the thermal and thermooxidative degradation of poly(3-hexyl thiophene). Thermochim. Acta, v. 499, p. 34-39, 2010.

VYAZOVKIN, S.; SBIRRAZZUOLI, N. Isoconversional Kinetic Analysis of Thermally Stimulated Processes in Polymers. Macromol. Rapid Commun., v. 27, p. 1515-1532, 2006. 